\title{
Brain-Behavior Participant Similarity Networks Among Youth and Emerging Adults with Schizophrenia Spectrum, Autism Spectrum, or Bipolar Disorder and Matched Controls
}

\author{
Laura Stefanik'1,2,3, Lauren Erdman ${ }^{4,5}$, Stephanie H Ameis 1,3,5,6, George Foussias ${ }^{1,2,3,6}$, Benoit H Mulsant ${ }^{1,3,6}$, \\ Tina Behdinan ${ }^{7}$, Anna Goldenberg ${ }^{4,5}$, Lauren J O'Donnell ${ }^{8}$ and Aristotle N Voineskos ${ }^{*, 1,2,3,6}$ \\ IInstitute of Medical Science, Faculty of Medicine, University of Toronto, Toronto, ON, Canada; ${ }^{2}$ Slaight Family Centre for Youth in Transition, Centre \\ for Addiction and Mental Health, Toronto, ON, Canada; ${ }^{3}$ Campbell Family Mental Health Research Institute, Centre for Addiction and Mental \\ Health, Toronto, ON, Canada; ${ }^{4}$ Department of Computer Science, University of Toronto, Toronto, ON, Canada; ${ }^{5}$ The Hospital for Sick Children, \\ Toronto, ON, Canada; ${ }^{6}$ Department of Psychiatry, University of Toronto, Toronto, ON, Canada; ${ }^{7}$ Schulich School of Medicine \& Dentistry, The \\ University of Western Ontario, London, ON, Canada; ${ }^{8}$ Brigham and Women's Hospital and Harvard Medical School, Boston, MA, US
}

\begin{abstract}
There is considerable heterogeneity in social cognitive and neurocognitive performance among people with schizophrenia spectrum disorders (SSD), autism spectrum disorders (ASD), bipolar disorder (BD), and healthy individuals. This study used Similarity Network Fusion (SNF), a novel data-driven approach, to identify participant similarity networks based on relationships among demographic, brain imaging, and behavioral data. TI-weighted and diffusion-weighted magnetic resonance images were obtained for 174 adolescents and young adults (aged 16-35 years) with an SSD $(n=51)$, an ASD without intellectual disability $(n=38)$, euthymic BD $(n=34)$, and healthy controls $(n=5 \mathrm{I})$. A battery of social cognitive and neurocognitive tasks were administered. Data integration, cluster determination, and biological group formation were then obtained using SNF. We identified four new groups of individuals, each with distinct neural circuitcognitive profiles. The most influential variables driving the formation of the new groups were robustly reliable across embedded resampling techniques. The data-driven groups showed considerably greater differentiation on key social and neurocognitive circuit nodes than groups generated by diagnostic analyses or dimensional social cognitive analyses. The data-driven groups were validated through functional outcome and brain network property measures not included in the SNF model. Cutting across diagnostic boundaries, our approach can effectively identify new groups of people based on a profile of neuroimaging and behavioral data. Our findings bring us closer to disease subtyping that can be leveraged toward the targeting of specific neural circuitry among participant subgroups to ameliorate social cognitive and neurocognitive deficits.

Neuropsychopharmacology (2018) 43, I I80-1 I88; doi:I0.1038/npp.20 I7.274; published online 6 December 2017
\end{abstract}

\section{INTRODUCTION}

Schizophrenia spectrum disorders (SSDs), autism spectrum disorders (ASD), and bipolar disorder (BD) exact enormous personal, social, and economic costs, and significantly impact quality of life and functional independence (Lazoff et al, 2010; Saha et al, 2005; Schaffer et al, 2006). All of these disorders are considered neurodevelopmental in origin, although ASDs typically onset earliest in life. Situated at a crucial phase of life, youth and emerging adults with mental illness experience great difficulty transitioning to independence, often due to impairment in functioning. Identification of brain-behavior relationships in those experiencing

* Correspondence: Dr AN Voineskos, Slaight Family Centre for Youth in Transition, Centre for Addiction and Mental Health, 250 College Street, Toronto, ON M5T IR8, Canada, Tel: +(4I6) 535-850 I ext. 33977, Fax: +(416) 260-4I62, E-mail: aristotle.voineskos@camh.ca

Received 8 August 2017; revised 6 October 2017; accepted 27 October 2017; accepted article preview online 6 November 2017 considerable impairment, as well as those who are less impaired, could accelerate therapeutic innovation and reduce disability at this critical developmental life stage.

The examination of social cognitive and neurocognitive impairment in those with an ASD, SSD, or BD is supported by a large body of literature citing the substantial overlap in behavioral dysfunction among these disorders (MartinsJunior et al, 2011; Millan et al, 2012). There is less overlap for instance in those with a unipolar affective illness (even with psychosis) and an SSD (Reichenberg, 2010). However, within-diagnostic heterogeneity can sometimes lead to even greater differences in cognition among those with the same diagnosis than between different diagnoses. Furthermore, among those with a diagnosis, performance may be no different than a person without mental illness.

Neuroimaging studies have also identified both similar and different brain circuitry vulnerability across these disorders. Abnormalities in these structures are often replicated, but recent large $\mathrm{n}$ studies by the ENIGMA Network suggest effect sizes are small likely due to heterogeneity. Biomarker 
identification in subgroups of individuals relevant for social cognitive and neurocognitive performance may be facilitated by cutting across disorders, including control participants, and using data integration approaches.

A recent study (Clementz et al, 2016) demonstrated the potential of a transdiagnostic clustering approach by characterizing neurobiological variation in relation to neurocognitive performance across individuals with schizophrenia, schizoaffective or bipolar disorder. Such an approach has yet to be studied for social cognitive performance, for which there is considerable overlap among those with an SSD or ASD. In addition, understanding potential overlap with healthy control populations, as in the $\mathrm{RDoC}$ approach, may enhance knowledge of the continuum of brain-behavior relationships.

In the present study, we used a novel approach known as Similarity Network Fusion (SNF) (Wang et al, 2014), which identifies participant similarity networks within and across data types. Our aims were to: (1) identify the profiles, reliability, and validity of the new data-driven groups across participants with an SSD, ASD, BD or matched controls based on the final SNF matrix, and (2) determine how SNF, diagnostic, and dimensional analyses compare on key variables identified in our model that have also been implicated in prior neuroimaging studies of SSDs, ASD, or BD. We hypothesized (Ameis and Catani, 2015; Behdinan, 2015; Voineskos et al, 2013), that we would discover new and robust participant similarity networks cutting across our original diagnostic categories revealing shared and divergent neural circuitsocial cognitive (or neurocognitive) impairments. We also anticipated that these groups would be distinguished with larger effect sizes in higher-order brain circuitry and cognitive functions than have typically been shown in either diagnostic comparisons or dimensional analyses.

\section{MATERIALS AND METHODS}

\section{Study Participants}

A total of 174 participants (SSDs, $N=51$; ASD, $N=38$; BD, $N=34$; controls, $N=51$ ) in this study were recruited through the Slaight Family Centre for Youth in Transition at the Centre for Addiction and Mental Health (CAMH) in Toronto, Canada. All participants provided voluntary written informed consent after having received a complete description of the study which was approved by the CAMH research ethics board. SSD, ASD, and BD participants were included if they were aged 16 to 35 years and stable with no psychiatric hospitalizations, changes in medication dose or type, or decrement in functioning or support level in the last 30 days. In addition, participants with $\mathrm{BD}$ were required to be euthymic based on Hamilton Rating Scale for Depression (HRSD) (Hamilton, 1980) and Young Mania Rating Scale (YMRS) (Young et al, 1978) scores of 10 or below. The Structured Clinical Interview for DSM-IV-TR (SCID) Axis I Disorders was administered by a trained interviewer to participants with a DSM-IV diagnosis of schizophrenia, schizoaffective disorder, psychotic disorder not otherwise specified, and bipolar disorder as well as healthy controls to confirm diagnostic eligibility. The Autism Diagnostic Observation Schedule-II (ADOS-II) (Lord et al, 2000) was administered by a child and youth psychiatrist (SHA) to confirm diagnostic eligibility of verbal ASD without intellectual disability. All ratings took into consideration a review of the participants' electronic health record and a consultation with each participant's treating psychiatrist.

Exclusion for all participants included: a history of substance use disorder (other than tobacco) in the past 6 months, a positive urine toxicology screen, contraindications for MRI, a concomitant major medical illness, a neurologic illness affecting the central nervous system, or a head trauma resulting in loss of

Table I Demographic and Clinical Characteristics of Adolescents and Young Adults with ASD, BD, SSD, and Healthy Controls (N= I74)

\begin{tabular}{|c|c|c|c|c|c|c|c|c|c|c|}
\hline \multirow[t]{2}{*}{ Characteristic } & \multicolumn{8}{|c|}{ Group } & \multicolumn{2}{|c|}{ Analysis } \\
\hline & \multicolumn{2}{|c|}{ Controls $(N=51)$} & \multicolumn{2}{|c|}{ BD $(N=34)$} & \multicolumn{2}{|c|}{$\operatorname{ASD}(N=38)$} & \multicolumn{2}{|c|}{$\operatorname{SSD}(N=5 I)$} & $\mathbf{F}$ & $p$ \\
\hline Age (years) & 24.20 & 4.24 & 26.41 & 4.56 & 22.55 & 4.64 & 26.49 & 4.81 & 6.71 & $<0.01$ \\
\hline Education (years) & 15.43 & 1.77 & 14.65 & 1.70 & 13.89 & 2.95 & $|3.5|$ & 1.91 & 9.74 & $<0.001$ \\
\hline \multirow[t]{2}{*}{ Gender (female) } & 27 & 53 & 15 & 44 & 12 & 31 & 19 & 37 & 4.74 & 0.19 \\
\hline & $N$ & & $N$ & & $N$ & & $N$ & & & \\
\hline Handedness & $48 \mathrm{R}, 3 \mathrm{~L}, 0 \mathrm{~A}$ & & $3 \mid R, 2 L, O A$ & & $32 \mathrm{R}, 4 \mathrm{~L}, 2 \mathrm{~A}$ & & $4 I R, 7 L, 3 A$ & & - & - \\
\hline BPRS & - & - & - & - & - & - & 28.72 & 6.83 & - & - \\
\hline HRSD & - & - & 4.82 & 2.49 & - & - & - & - & - & - \\
\hline
\end{tabular}

Abbreviations: ASD, autism spectrum disorder; BD, bipolar disorder, BPRS, Brief Psychiatric Rating Scale; HRSD, The Hamilton Rating Scale for Depression; RBS, The Repetitive Behavior Scale; SSD, schizophrenia spectrum disorder; SRS, Social Responsiveness Scale; YMRS, Young Mania Rating Scale.

Handedness: $R=$ right, $L=$ left, $A=$ ambidextrous. 
consciousness for more than thirty minutes. Further exclusions for controls included the presence of any current DSM-IV disorder, or any past DSM-IV disorder with the exception of specific phobia or adjustment disorder. The demographic characteristics of the sample are summarized in Table 1 and the psychotropic medication information is provided in Supplementary Table S1.

To further characterize the clinical status of each disease group, the Brief Psychiatric Rating Scale (BPRS-18) (Overall and Gorham, 1962) was administered to participants with SSDs to measure the severity of positive, negative, and affective symptoms. The Repetitive Behaviors Scale - Revised (RBS-R) (Lam and Aman, 2007) and Social Responsiveness Scale (SRS) (Bölte et al, 2008) were administered to participants with ASD to provide quantitative severity measures of repetitive behaviors and social responsiveness. The HRSD and YMRS were administered to participants with BD to verify euthymic status (see above).

\section{Social Cognitive and Neurocognitive Assessments}

The Awareness of Social Inference Test - Revised (TASIT) Parts I, II, and III were used to assess social cognitive deficits. The TASIT I is used to assess a participant's ability to gauge emotional expressions, TASIT II assesses the evaluation of social interactions at a minimal level, and the TASIT III assesses the evaluation of social interactions at an enriched level, including detection of lies and sarcasm (McDonald et al, 2003).

Neurocognition was assessed using the MATRICS Consensus Cognition Battery (MCCB) in those with an SSD, BD, and healthy controls. For participants with ASD the Wechsler Adult Intelligence Scale (WAIS) was administered. Subtests assessing working memory (spatial span) and processing speed (coding task) were identified as shared across the MATRICS and WAIS, and the scores were standardized across batteries.

\section{MRI}

All brain imaging for the present study was performed on a 3 Tesla Discovery MR750 (General Electric) system at CAMH using an 8-channel head coil. Detailed acquisition parameters for the anatomical and diffusion scans are provided in the supplemental material. All diffusion scans were acquired with 60 non-collinear directions, using $2 \mathrm{~mm}$ isotropic voxels.

T1-weighted images were processed on the same workstation using FreeSurfer's fully automatic structural imaging software package (version 5.3.0) (http://surfer.nmr.mgh.harvard.edu/). Both cortical thickness and subcortical volumes were then processed using the ENIGMA processing protocol (http:// enigma.usc.edu/) and values were normalized using the standardNormalization function in the SNFtool R package. For white matter analyses, tensors and fiber tracts were generated in 3D Slicer (Fedorov et al, 2012) via the SlicerDMRI project (dmri.slicer.org; Norton et al, 2017) and an automated method was used for tractography segmentation (dmri.slicer. org/whitematteranalysis). This fiber clustering method has been previously applied in a number of studies (O'Donnell et al, 2009, 2017; Propper et al, 2010; Voineskos et al, 2009, 2010, 2012; Whitford et al, 2010; Zhang et al, 2016) and has been validated against expert tract selection (Voineskos et al, 2009). An advantage of the method is that it enables a study-specific anatomical definition of tracts of interest that are relevant for testing a particular hypothesis. See supplemental material for more detail on atlas generation and measurement (fractional anisotropy and mean diffusivity) from the 19 white matter tracts segmented.

\section{Data Integration and Cluster Determination}

R statistical software v.3.3.2 (www.r-project.org) with the SNFtool package v.2.2 was installed (https://cran.r-project. org/web/packages/SNFtool/) to run the SNF algorithm. SNF methods have been previously described (Wang et al, 2014) and are freely available (http://compbio.cs.toronto.edu/SNF/). Briefly, SNF combines a number of data types for a given set of patients, constructs a similarity network for each data source, and iteratively updates and integrates these networks using a nonlinear fusion method into one global network. More details regarding the mathematical underpinnings of the algorithm and its key advantages are outlined in the supplemental methods.

Performance of the SNF algorithm was assessed by testing the stability of the model, ranking variables using Normalized Mutual Information (NMI), and annotating the clusters to match individual participants to their cluster assignment. The rankfeaturesbyNMI function calculates the relative contribution of each variable in driving the patient groups. NMI is a measure of similarity between a clustering outcome defined by all of the data combined and a clustering outcome defined by the single feature of interest.

The SNFtool package was customized to integrate demographic, social cognitive, neurocognitive, cortical thickness, subcortical volume, and diffusion tensor imaging tractography data ( $n=137$ variables). For a full variable list see Supplementary Table S2. Based on the number of participants in our sample, the optimal clustering range for the model was first determined using the guideline for $K$ ( participant $n / 10$ ) (Wang et al, 2014), then confirmed using a grid search function whereby each value for variable $\mathrm{K}$ between the recommended range of $10-30$ was applied to the estimatenumberofcluster function.

\section{Model Stability and Reliability}

Once hyperparameter optimization was complete and a fused network was produced, a two-step approach was used to assess the stability of the model and reliability of the new data-driven groups. First, an embedded robust core clustering function sampled $80 \%$ of participants $(N=139)$ for a total of 100 random draws to determine the stability of participant cluster membership. Second, an embedded replication technique was applied whereby $50 \%$ of participants $(N=87)$ were randomly sampled for a total of 100 random draws. Data integration, cluster determination, and biological group formation were obtained for each draw. The top 15 variables ranked by NMI were compared between the primary analysis and the replication groups.

\section{Brain Circuit and Behavior Comparisons Among the New Data-Driven Groups}

Welch's ANOVA was used to compare the 15 most influential variables (out of a total of 137 demographic, 
neuroimaging and cognitive variables) between the new SNF-derived groups followed by multiple comparison correction. Selecting the top 15 ensures a focus on variables with medium or large effect sizes. This test was used due to lack of homogeneity of variance. Results were validated using Kruskal-Wallis tests and post hoc testing followed, when applicable, using the Dunn test. Fisher's exact test $/ \chi^{2}$ test were used for categorical variables. These analyses were conducted for a second time in a more restricted age-range (16-29) with no age range skew due to the slight upward skew of the schizophrenia and bipolar groups in the full sample.

\section{Independent Validation of Data-Driven Groups}

In order to examine whether the data-driven groups provide clinically meaningful information at a real-world level, the total score from the Birchwood Social Functioning Scale, which was employed in the SSD participants and healthy controls to assess social function, was compared among the data-driven groups. The Mayer-Salovey-Caruso Emotional Intelligence Test (MSCEIT) total score, which was employed to assess emotional intelligence, was also compared among the data-driven groups.

In addition, data-driven groups were validated using new neuroimaging data. The graph theory metric of global efficiency for structural networks was evaluated using cortical thickness data. A permutation test was run to compare the identified distribution of differences to those if the structure correlations were randomized.

\section{RESULTS}

\section{Rank of Variable Importance Contributing to Formation of New Groups}

Of the 137 neuroimaging, behavioral, and demographic variables in the model, the top 15 ranked by NMI are shown in Table 2 with standardized mean values for each group. The most influential demographic variable was age. The most influential neuroimaging variables were volume of the right pallidum, FA of the right arcuate fasciculus and right cingulum bundle, and thickness of the superior frontal gyrus and right inferior parietal gyrus, and the most influential cognitive variables were processing speed and TASIT 3 score.

\section{Composition and Reliability of New Data-Driven Groups}

The fused network revealed four new participant similarity network groups. For diagnostic and clinical properties of the new groups see Figure 1 and Supplementary Table S3 respectively. Group 1 was an ASD primary group with SSD secondary; Group 2 was an SSD primary group with minor distribution of the other groups; Group 3 was comprised of approximately $50 \%$ healthy controls, with distribution of the other three groups, while Group 4 had sizeable proportion of each of the four diagnostic groups.

On average, $80 \%$ of the top 15 variables overlapped across analyses speaking to the stability of the brain-circuit cognitive profiles. See Supplementary Table S4 for the rank order of the top 15 variables based on their average NMI score across resampling.

\section{Brain Circuit-Cognitive Profiles of the Data-Driven Groups}

We found significant differences in: age (Welch's $F$ $\left.(3,68)=33.36, \quad p<0.001, \quad \eta^{2}=0.40\right), \quad$ in right pallidum (Welch's $\mathrm{F}(3,66)=30.16, p<0.001, \eta^{2}=0.32$ ) and hippocampus volumes (Welch's $\mathrm{F}(3,69)=18.15, \quad p<0.001$, $\left.\eta^{2}=0.23\right)$, in cortical thickness of the superior frontal gyrus (Welch's $\left.\mathrm{F}(3,71)=11.14, p<0.001, \eta^{2}=0.17\right)$, and right inferior parietal gyrus (Welch's $\mathrm{F}(3,68)=7.43, p<0.001$, $\eta^{2}=0.14$ ), in right arcuate fasciculus (Welch's $\mathrm{F}$ $\left.(3,66)=11.79, p<0.001, \eta^{2}=0.19\right)$, and bilateral cingulum bundle FA (Welch's $\left.\mathrm{F}(3,65)=18.93, p<0.001, \eta^{2}=0.25\right)$, and

Table 2 Top 15 Variables by NMI Score: Mean Values Per Data-Driven Group (z-Scored) and Collapsed When Bilateral Representation Occurred

\begin{tabular}{|c|c|c|c|c|c|c|}
\hline Rank & Variable & NMI Score & Group I & Group 2 & Group 3 & Group 4 \\
\hline I & Age & 0.13 & -0.89 & 1.22 & -0.33 & 0.11 \\
\hline 2 & Processing speed & 0.13 & -0.66 & -0.28 & 0.50 & -0.07 \\
\hline 3 & Volume right pallidum & 0.12 & 0.64 & 0.09 & -0.76 & 0.41 \\
\hline 4 & FA right arcuate fasciculus & 0.11 & -0.72 & -0.28 & -0.13 & 0.53 \\
\hline 5 & FA right cingulum bundle & 0.11 & -0.67 & -0.45 & -0.17 & 0.62 \\
\hline 8 & Thickness right inferior parietal gyrus & 0.10 & 0.38 & -0.83 & 0.09 & 0.13 \\
\hline 9 & Volume right putamen & 0.10 & 0.93 & -0.48 & -0.49 & 0.30 \\
\hline 10 & Volume right amygdale & 0.10 & 0.93 & -0.47 & -0.51 & 0.32 \\
\hline 11 & Volume of the right hippocampus & 0.09 & 0.68 & -0.38 & -0.51 & 0.37 \\
\hline
\end{tabular}

Note: Working memory NMI score $=0.03$, Part I total correct - TASIT NMI score $=0.02$, and Part 2 total correct - TASIT NMI score $=0.03$. 

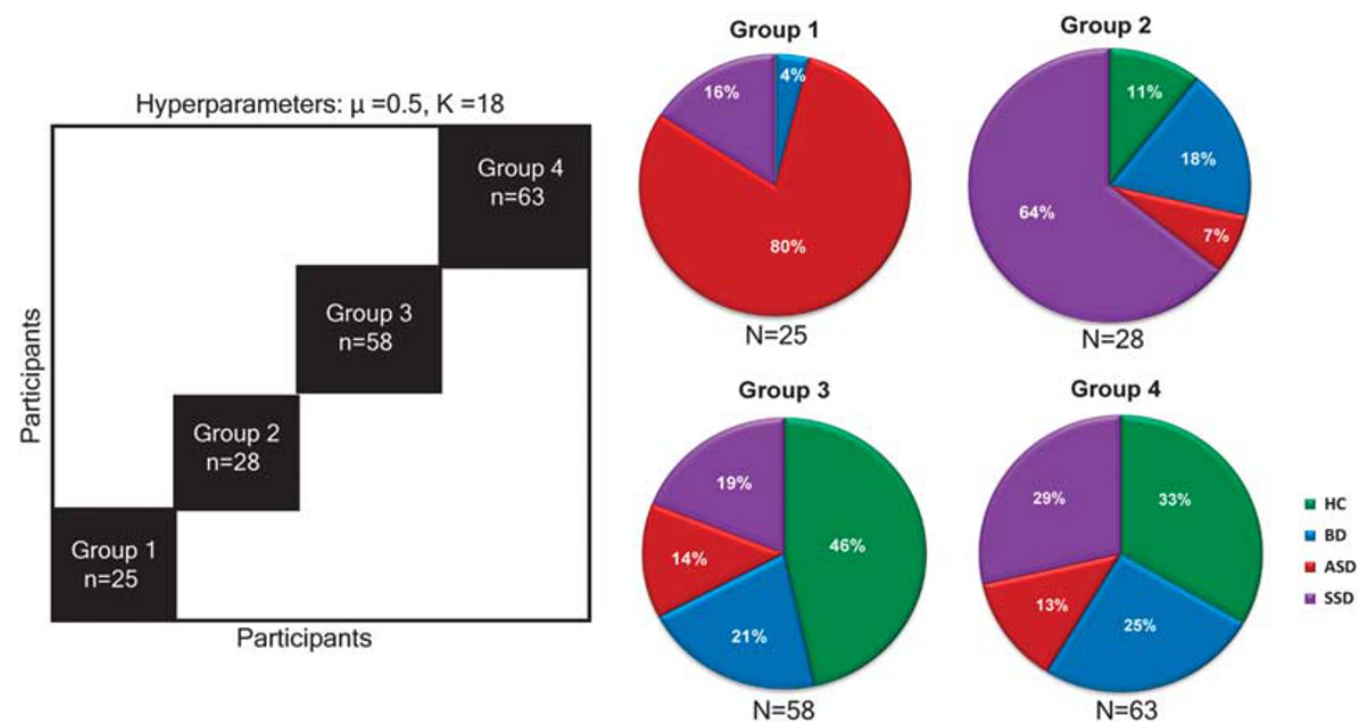

Figure I Outline representation of the fused matrix and the diagnostic composition of the data driven groups. Four groups of participants identified using Similarity Network Fusion with different profiles of brain-behavior relationships that cut across conventional DSM diagnostic categories.

in processing speed (Welch's $\mathrm{F}(3,75)=14.21, p<0.001$, $\eta^{2}=0.16$ ), and TASIT3 (Welch's $\mathrm{F}(3,65)=16.58, p<0.001$, $\eta^{2}=0.25$ ) scores. All of these results survive multiple comparison correction. Neural and cognitive profiles for each of the new data-driven groups on some of these influential variables can be seen in Figures 2 and 3. Post hoc analyses in the 16-29 year age range showed similar results (Supplementary Information).

\section{Brain Circuit-Cognitive Profiles by Diagnostic and Dimensional Comparisons Respectively}

Our SNF results are a powerful contrast to the same plots being generated by diagnostic group (see Supplementary Figure S1 and S2). While FA of the cingulum bundle (Welch's $\left.F(3,84)=4.67, p<0.01, \eta^{2}=0.10\right)$ and arcuate fasciculus remain the lowest in the ASD group (Welch's F $\left.(3,88)=3.76, \quad p=0.01, \quad \eta^{2}=0.09\right)$, and superior frontal (Welch's $\mathrm{F}(3,92)=3.10, p=0.03, \eta^{2}=0.04$ ) and right inferior parietal (Welch's $\mathrm{F}(3,91)=1.91, p=0.13, \eta^{2}=0.03$ ) cortical thickness the lowest in the SSD group, these differences are not nearly as pronounced and in some cases not statistically significant between groups, with small effect sizes. When participants were separated into quartiles based on TASIT Part III scores, ie., a dimensional approach, effect sizes were small and results did not survive multiple comparison correction. See Supplementary Table S5 for effect sizes across comparison types.

\section{Independent Validation of the Data-Driven Groups}

We found significant differences in Birchwood total score (Welch's $t=61.84, p<0.0001, d=0.82$ ) across groups, with Groups 1 and 2 having significantly lower scores (indicating poorer functioning) $(M=140.88, \mathrm{SD}=24.96)$ than Groups 3 and $4(M=160.23, \mathrm{SD}=23.01)$.

Groups 1 and 2 also had significantly lower MSCEIT scores (indicating poorer emotional intelligence) than
Groups 3 \& 4 (Welch's $\left.\mathrm{F}(3,18)=4.85, p=0.01, \eta^{2}=0.10\right)$. Groups 2 and 4 showed reductions in nodal global efficiency relative to Group 1 ( $p=0.026$ and 0.041 respectively). There was also a significant reduction in global efficiency for Group 4 relative to Group 3( $p=0.031)$. These significant differences survived a Bonferroni multiple testing correction. More detail on the analytic methodology is provided in the Supplementary Material.

\section{DISCUSSION}

Following the acquisition of neuroimaging, social cognitive, neurocognitive, and demographic data in adolescents and young adults with SSD, ASD, or BD, and typically developing ('healthy') controls, we applied SNF to identify new participant similarity networks. We identified four new groups of individuals, each with different neural circuitcognitive profiles. In both the 16-35 and16-29 age range analyses, the neuroimaging variables with the highest NMI values, and thereby those of greatest importance in the integrated clustering matrix, were: the right cingulum and arcuate fasciculus (two higher-order white matter tracts), cortical thickness (fronto-parietal regions), and subcortical volume (hippocampus/amygdala and striatal). These structures are consistent with known social cognitive and neurocognitive brain circuit nodes of the right frontoparietal mirror neuron system (MNS), and cortical midline circuit (Carr et al, 2003; Mancuso et al, 2011). The datadriven groups showed considerably greater differentiation, as measured via effect size, on these neuroimaging measures than the original categorical (diagnostic) groups or groups from the dimensional analyses. The validity of these new groups was independently demonstrated through differences in social function and brain network organization.

Group 1 revealed deficits in white matter tracts and nodes of the MNS and limbic system including the right arcuate fasciculus, bilateral cingulum bundle, and the left uncinate fasciculus. These findings are consistent with robust evidence 

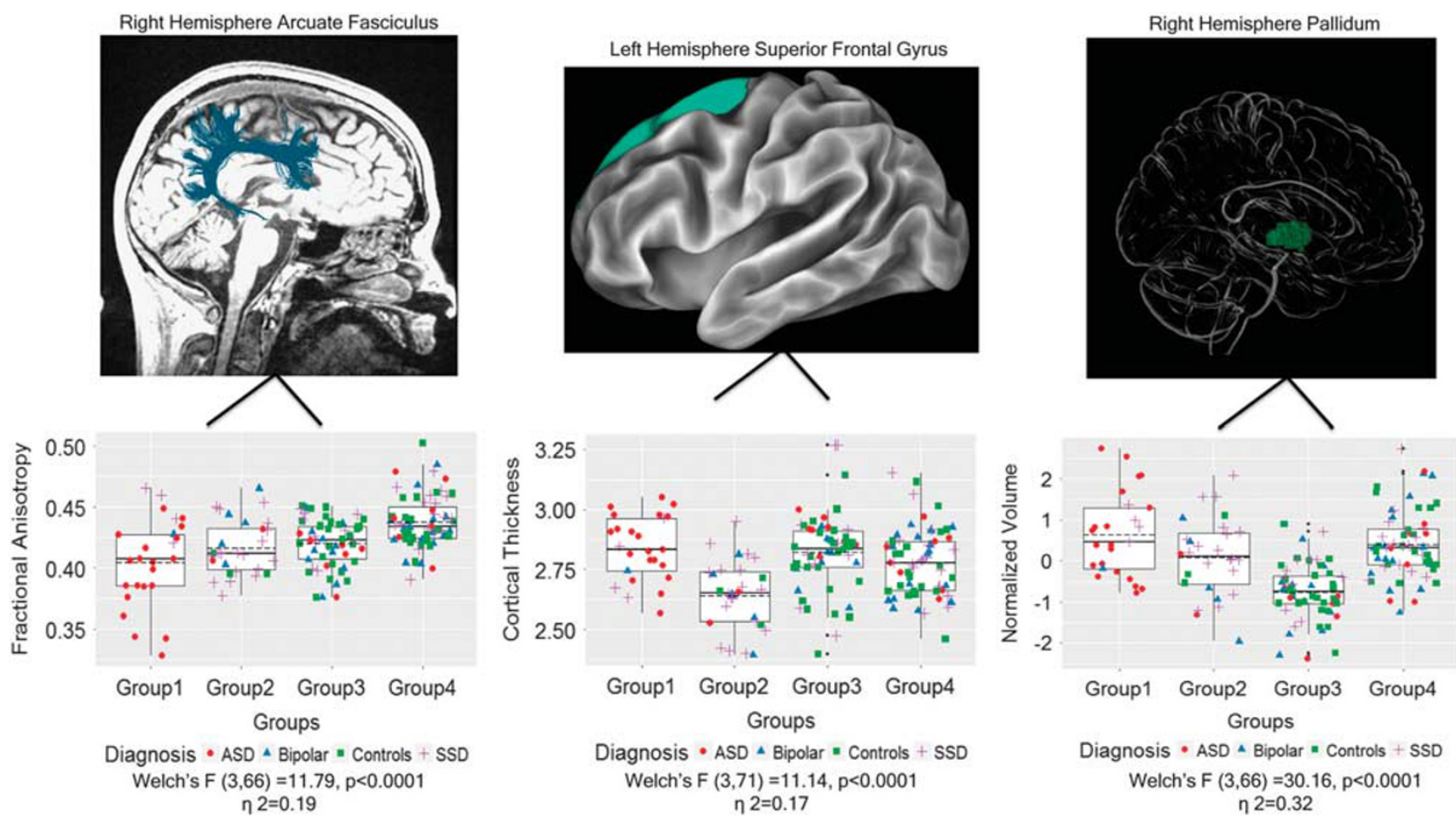

Figure 2 Distinct neural circuit profiles of the data-driven groups. Evidence of distinct brain-circuit profiles for the data-driven groups. Pictured are the most influential regions or tract for each neuroimaging index (left to right): differing fractional anisotropy profiles of groups for the right arcuate fasciculus, differing cortical thickness profiles of groups for left superior frontal gyrus, and differing subcortical volume profiles of groups for the right pallidum. Note: median (solid) and mean (broken line) scores depicted.
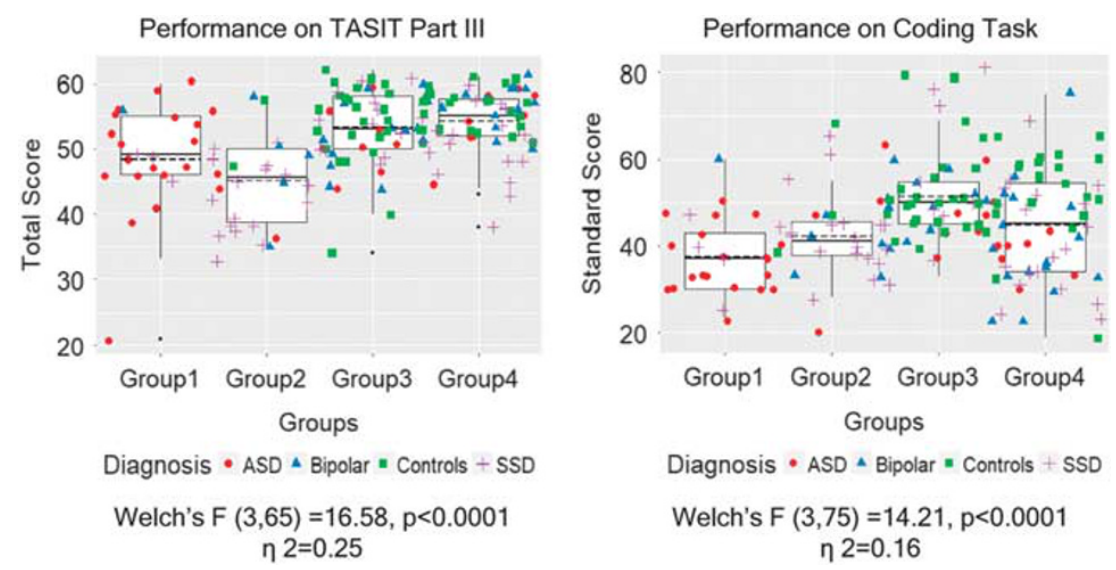

Figure 3 Distinct cognitive profiles of the data-driven groups. Evidence of distinct cognitive profiles for the data-driven groups. Pictured are the most influential cognitive measures (left to right): differing social cognitive profiles of groups for the TASIT Part III and differing neurocognitive profiles for the Coding Task (Performance Speed Index). Note: median (solid) and mean (broken line) scores depicted.

pointing to alterations in white matter tracts during early development in ASD (that are also present in adult samples) for the arcuate and uncinate fasciculi (Ameis and Catani, 2015). Deficits in these same white matter tracts are also seen in individuals with SSD, which is the second-most prevalent diagnostic category in this group. Group 2, characterized by SSD as the most common category, also demonstrated impairment in these white matter tracts but not to the same extent as Group 1. However, Group 2 showed the greatest impairments in social cognitive and neurocognitive performance, along with the greatest cortical thickness reductions among all groups in a number of mentalizing and executive function nodes. In contrast, Group 1 showed the greatest cortical thickness among all four groups. A study design using traditional categorical diagnostic groups may not have been able to parse this brain-behavior heterogeneity. In addition, our diagnostic group comparisons showed only small effect size among the brain circuitry and structures most influential (based on NMI score and rank) in separating the new groups, consistent with large $\mathrm{n}$ studies from the ENIGMA work group (van Erp et al, 2016; Kelly et al, 2017). Further, the small effect sizes when using a dimensional approach (based on quartiles of social cognitive performance) indicate that the integration of multi- 
dimensional data is critical for both biological group formation and detection of brain-behavior relationships.

We suggest that Group 1 and Group 2 may represent 'tipping point' subsamples (Cuthbert and Insel, 2013), as per the RDoC model, with extremes of neural substrates (low FA and high cortical thickness in Group 1 vs low cortical thickness in Group 2). Given the genetic overlap between SSDs and ASDs some with an SSD might have generalized cerebral cortical enlargement, exemplified by those who were similar to ASDs in Group 1. Our approach also identified those individuals with an SSD with statistically lower tractspecific FA, and greater neurobiological similarity to those with an ASD in Group 1, and those with an ASD or BD with severe cortical thickness reductions more commonly identified in SSDs in Group 2. The high cortical thickness in Group 1 may be suggestive of brain growth abnormalities-a feature of some patients with ASD (Courchesne and Pierce, 2005). The validation approach using a network efficiency measure supports the divergence in cortical thickness seen in Group 1 (highest thickness in key social and neurocognitive nodes) relative to Group 2 (lowest thickness). The inefficiencies in Groups 2 and 4 suggest the network organization may lead to a reduced capacity to integrate information required for effective social and neurocognitive task performance.

Nearly $50 \%$ of the participants in Group 3 were healthy, but the remainder were participants with ASD, SSD, or BD. These individuals may represent patient participants who show no differences on cognitive or neuroimaging measures from controls, and may represent a higher-functioning and resilient patient group. Our findings supports those of Clementz and colleagues (Clementz et al, 2016) who identified a higher-functioning psychosis biotype for whom measures of cognitive control did not differ between the cluster members and healthy controls. A total of $20-40 \%$ of individuals with schizophrenia spectrum disorders have minor cognitive impairment compared to normative data (Rund et al, 2007; Vaskinn et al, 2009; Weickert et al, 2000). In euthymic $\mathrm{BD}$, the literature suggests that high functioning $\mathrm{BD}$ does not differ from low functioning $\mathrm{BD}$ based on clinical variables (Martinez-Aran et al, 2007), but rather the number of medications and the level of cognitive function, which was the case in our BD participants. Group 4 consisted mostly of participants with SSD or BD with relatively preserved social cognition but more impaired in processing speed compared to Group 3. A number of studies have documented qualitatively similar cognitive deficits in SSD and $\mathrm{BD}$, with a general consensus that deficits are milder in patients with BD (Czobor et al, 2007; Green, 2006; Schretlen et al, 2007; Vöhringer et al, 2013). It is possible that the overlap in impairment captures why these two diagnostic groups are most represented in Group 4.

The post hoc analysis was conducted to address the relative importance of age in the SNF matrix for the primary analysis. The upper boundary of 29 years of age was selected to maximize our sample size while eliminating the statistically significant mean age differences between the diagnostic groups that were skewed toward older BD and SSD participants. Moreover, the emerging adulthood literature has recognized that while the focus of the developmental period is 18-25 years of age, the upper age boundary is flexible and spans up to 29 years of age (Arnett, 2004; Arnett et al, 2014) or even into one's early $30 \mathrm{~s}$ (Trible, 2015). Data from this analysis provided preliminary evidence for the stability of the structural markers of impairment for social cognition across multiple iterations of the model and showed that qualitative similarities in both the diagnostic distribution and brain-behavior profiles of the groups persisted irrespective of the influence of age. This new analysis primarily eliminated participants with an SSD $(n=17)$, and a small number of BD and HC individuals. Group 1 and Group 2 show nearly exactly the same mean $z$-scores on both social cognitive and neurocognitive performance. This post hoc analysis, therefore, even more clearly demonstrates that these two more impaired groups, while showing similar neurocognitive and social cognitive difficulties, show completely different neural substrates of that impairment.

Our study has some limitations that should be considered. First, most of the participants with ASD, SSD, or BD were medicated and this was not taken into consideration in the SNF model. However, post hoc analyses examining the relationship between mean chlorpromazine index values (for the data-driven groups) and the top NMI variables revealed that these variables are not significantly correlated. Second, we have not tested the longitudinal stability of our groups. Third, while we cannot completely rule out the effects of age in our model, the post hoc analysis in 16-29 year olds provides compelling evidence that when age distribution is similar, the participant groups are driven more by stable brain and behavior markers than age. We also acknowledge that with an even larger sample size we may have had the power to detect other relationships between neural circuit structure and social cognition, and despite our re-sampling/ reliability results, our findings require validation on independent datasets.

The clinical utility of SNF addresses the need for advancements in precision medicine in psychiatry. While the original paper introducing the method was applied to genomic data (Wang et al, 2014), SNF is not specifically suited to cancer genomics and has already been applied to different data types, including successful application to neuroimaging data ( $\mathrm{Li}$ et al, 2015). The application of transdiagnostic data integration algorithms like SNF take advantage of the heterogeneity in a clinical sample to identify groups of individuals with greater homogeneity in brain circuit and behavioral measures. It can also serve to differentiate among individuals with similar behavioral impairments, but different brain circuit impairments. Analyzing the structural correlates of social cognitive deficits has the potential to identify intervention targets. The goal of our work in this field is to provide clear targets for intervention that may cut across disorders with demonstrated relevance for social functioning in the community. Two randomized controlled trials by our group showed the potential for this approach either in study design or therapeutic efficacy (Ameis et al, 2017; Barr et al, 2013).

Independent validation of the SNF approach and the neural circuit structure-performance relationships reported in our analyses may ultimately lead to the implementation of clustering and clinical predictive algorithms in a clinical setting with a number of disease groups. Improvements in etiological subtyping would also have larger implications for therapeutic innovation in social cognitive and neurocognitive domains. 


\section{FUNDING AND DISCLOSURE}

LS is funded by the Ontario Graduate Scholarship Program (Faculty of Medicine, University of Toronto). SHA is funded by the Ontario Mental Health Foundation and Canadian Institutes of Health Research. BHM has received grant support from the National Institute of Mental Health, the Canadian Institutes for Health Research, Brain Canada, the $\mathrm{CAMH}$ Foundation, and PCORI. He has also received free medications for NIH-funded trials from Eli-Lilly, BristolMyers-Squibb, and Pfizer. He owns General Electric stock (less than \$5000). ANV is funded by the Canadian Institutes of Health Research, Ontario Mental Health Foundation, Brain and Behavior Research Foundation, and the National Institute of Mental Health (Grant Nos. R01MH099167 and R01MH102324). GF has served as an investigator on research sponsored by Medicure Inc., and Neurocrine Bioscience. He has also served on advisory boards for Hoffman-La Roche and Takeda, and received speaker's fees from Hoffman-La Roche, Lundbeck and Novartis. LJO is funded by the National Institutes of Health (Grant Nos. P41EB015902, P41EB015898, U01CA199459, R01MH074794, and R03NS088301). The remaining authors declare no conflict of interests.

\section{REFERENCES}

Ameis SH, Catani M (2015). Altered white matter connectivity as a neural substrate for social impairment in autism spectrum disorder. Cortex 62: 158-181.

Ameis SH, Daskalakis ZJ, Blumberger DM, Desarkar P, Drmic I, Mabbott DJ et al (2017). Repetitive transcranial magnetic stimulation for the treatment of executive function deficits in autism spectrum disorder: clinical trial approach. J Child Adolesc Psychopharmacol 27: 413-421.

Arnett JJ (2004). Emerging Adulthood: The Winding Road From The Late Teens Through The Twenties. Oxford University Press: Oxford, UK, 2006.

Arnett JJ, Žukauskienė R, Sugimura K (2014). The new life stage of emerging adulthood at ages 18-29 years: implications for mental health. Lancet Psychiatry 1: 569-576.

Barr MS, Farzan F, Rajji TK, Voineskos AN, Blumberger DM, Arenovich $\mathrm{T}$ et al (2013). Can repetitive magnetic stimulation improve cognition in schizophrenia? Pilot data from a randomized controlled trial. Biol Psychiatry 73: 510-517.

Behdinan T (2015). Dissecting shared and unique neural circuitry underlying negative symptoms, social cognition, and functional outcome in schizophrenia, MSc Thesis, University of Toronto, Toronto, ON.

Bölte S, Poustka F, Constantino JN (2008). Assessing autistic traits: cross-cultural validation of the social responsiveness scale (SRS). Autism Res 1: 354-363.

Carr L, Iacoboni M, Dubeau M-C, Mazziotta JC, Lenzi GL (2003). Neural mechanisms of empathy in humans: a relay from neural systems for imitation to limbic areas. Proc Natl Acad Sci USA 100: 5497-5502.

Clementz BA, Sweeney JA, Hamm JP, Ivleva EI, Ethridge LE, Pearlson GD et al (2016). Identification of distinct psychosis biotypes using brain-based biomarkers. Am J Psychiatry 173: 373-384.

Courchesne E, Pierce K (2005). Brain overgrowth in autism during a critical time in development: implications for frontal pyramidal neuron and interneuron development and connectivity. Int J Dev Neurosci 23: 153-170.

Cuthbert BN, Insel TR (2013). Toward the future of psychiatric diagnosis: the seven pillars of RDoC. BMC Med 11: 126.
Czobor P, Jaeger J, Berns SM, Gonzalez C, Loftus S (2007). Neuropsychological symptom dimensions in bipolar disorder and schizophrenia. Bipolar Disord 9: 71-92.

Erp TGM, van, Hibar DP, Rasmussen JM, Glahn DC, Pearlson GD, Andreassen OA et al (2016). Subcortical brain volume abnormalities in 2028 individuals with schizophrenia and 2540 healthy controls via the ENIGMA consortium. Mol Psychiatry 21: 547-553.

Fedorov A, Beichel R, Kalpathy-Cramer J, Finet J, Fillion-Robin J-C, Pujol S et al (2012). 3D Slicer as an image computing platform for the Quantitative Imaging Network. Magn Reson Imaging 30: 1323-1341.

Green MF (2006). Cognitive impairment and functional outcome in schizophrenia and bipolar disorder. J Clin Psychiatry 67(Suppl 9): 3-8-42.

Hamilton M (1980). Rating depressive patients. J Clin Psychiatry 41: 21-24.

Kelly S, Velzen L, van, Veltman D, Thompson P, Jahanshad N, Schmaal L et al (2017). White matter microstructural differences in major depression: meta-analytic findings from enigmaMDD DTI. Biol Psychiatry 81: S381.

Lam KSL, Aman MG (2007). The repetitive behavior scale-revised: independent validation in individuals with autism spectrum disorders. J Autism Dev Disord 37: 855-866.

Lazoff T, Zhong L, Piperni T, Fombonne E (2010). Prevalence of pervasive developmental disorders among children at the English Montreal School Board. Can J Psychiatry 55: 715-720.

Li G, Wang L, Gilmore JH, Lin W, Shen D (2015). Parcellation of infant surface atlas using developmental trajectories of multidimensional cortical attributes. Med Image Comput Comput Assist Interv, (2015) 9351: 543-550.

Lord C, Risi S, Lambrecht L, Cook EH, Leventhal BL, DiLavore PC et al (2000). The autism diagnostic observation schedule-generic: a standard measure of social and communication deficits associated with the spectrum of autism. J Autism Dev Disord 30: 205-223.

Mancuso F, Horan WP, Kern RS, Green MF (2011). Social cognition in psychosis: multidimensional structure, clinical correlates, and relationship with functional outcome. Schizophr Res 125: 143-151.

Martinez-Aran A, Vieta E, Torrent C, Sanchez-Moreno J, Goikolea J, Salamero $M$ et al (2007). Functional outcome in bipolar disorder: the role of clinical and cognitive factors. Bipolar Disord 9: 103-113.

Martins-Junior FE, Sanvicente-Vieira B, Grassi-Oliveira R, Brietzke E (2011). Social cognition and theory of mind: controversies and promises for understanding major psychiatric disorders. Psychol Neurosci 4: 347-351.

McDonald S, Flanagan S, Rollins J, Kinch J (2003). TASIT: A new clinical tool for assessing social perception after traumatic brain injury. J Head Trauma Rehabil 18: 219-238.

Millan MJ, Agid Y, Brüne M, Bullmore ET, Carter CS, Clayton NS et al (2012). Cognitive dysfunction in psychiatric disorders: characteristics, causes and the quest for improved therapy. Nat Rev Drug Discov 11: 141-168.

Norton I, Essayed I, Zhang F, Pujol S, Yarmarkovich A, Golby A et al (2017). SlicerDMRI: open source diffusion MRI software for brain cancer Research. Cancer Res 77: e101-e103.

O’Donnell LJ, Suter Y, Rigolo L, Kahali P, Zhang F, Norton I et al (2017). Automated white matter fiber tract identification in patients with brain tumors. NeuroImage Clin 13: 138-153.

O’Donnell LJ, Westin C-F, Golby AJ (2009). Tract-based morphometry for white matter group analysis. Neuroimage 45: 832-844.

Overall JE, Gorham DR (1962). The Brief Psychiatric Rating Scale. Psychol Rep 10: 799-812.

Propper RE, O’Donnell LJ, Whalen S, Tie Y, Norton IH, Suarez RO et al (2010). A combined fMRI and DTI examination of functional language lateralization and arcuate fasciculus structure: 
Effects of degree versus direction of hand preference. Brain Cogn 73: 85-92.

Reichenberg A (2010). The assessment of neuropsychological functioning in schizophrenia. Dialogues Clin Neurosci 12: 383-392.

Rund BR, Melle I, Friis S, Johannessen JO, Larsen TK, Midbøe LJ et al (2007). The course of neurocognitive functioning in firstepisode psychosis and its relation to premorbid adjustment, duration of untreated psychosis, and relapse. Schizophr Res 91: $132-140$.

Saha S, Chant D, Welham J, Mcgrath J (2005). A systematic review of the prevalence of schizophrenia. PLoS Med 2: e141.

Schaffer A, Cairney J, Cheung A, Veldhuizen S, Levitt A (2006). Community survey of bipolar disorder in Canada: lifetime prevalence and illness characteristics. Can J Psychiatry 51: 9-16.

Schretlen DJ, Cascella NG, Meyer SM, Kingery LR, Testa SM, Munro CA et al (2007). Neuropsychological functioning in bipolar disorder and schizophrenia. Biol Psychiatry 62: 179-186.

Trible H (2015). Emerging adulthood: defining the life stage and its developmental tasks, Thesis, James Madison university, Harrisonburg, VA.

Vaskinn A, Sergi MJ, Green MF (2009). The Challenges of ecological validity in the measurement of social perception in schizophrenia. J Nerv Ment Dis 197: 700-702.

Vöhringer PA, Barroilhet SA, Amerio A, Reale ML, Alvear K, Vergne D et al (2013). Cognitive impairment in bipolar disorder and schizophrenia: a systematic review. Front Psychiatry 4: 87.

Voineskos AN, Foussias G, Lerch J, Felsky D, Remington G, Rajji TK et al (2013). Neuroimaging evidence for the deficit subtype of schizophrenia. JAMA Psychiatry 70: 472.
Voineskos AN, Lobaugh NJ, Bouix S, Rajji TK, Miranda D, Kennedy JL et al (2010). Diffusion tensor tractography findings in schizophrenia across the adult lifespan. Brain 133: 1494-1504.

Voineskos AN, O’Donnell LJ, Lobaugh NJ, Markant D, Ameis SH, Niethammer $M$ et al (2009). Quantitative examination of a novel clustering method using magnetic resonance diffusion tensor tractography. Neuroimage 45: 370-376.

Voineskos AN, Rajji TK, Lobaugh NJ, Miranda D, Shenton ME, Kennedy JL et al (2012). Age-related decline in white matter tract integrity and cognitive performance: a DTI tractography and structural equation modeling study. Neurobiol Aging 33: 21-34.

Wang B, Mezlini AM, Demir F, Fiume M, Tu Z, Brudno M et al (2014). Similarity network fusion for aggregating data types on a genomic scale. Nat Methods 11: 333-337.

Weickert TW, Goldberg TE, Gold JM, Bigelow LB, Egan MF, Weinberger DR (2000). Cognitive impairments in patients with schizophrenia displaying preserved and compromised intellect. Arch Gen Psychiatry 57: 907-913.

Whitford TJ, Kubicki M, Schneiderman JS, O’Donnell LJ, King R, Alvarado JL et al (2010). Corpus callosum abnormalities and their association with psychotic symptoms in patients with schizophrenia. Biol Psychiatry 68: 70-77.

Young RC, Biggs JT, Ziegler VE, Meyer DA (1978). A rating scale for mania: reliability, validity and sensitivity. Br J Psychiatry 133: $429-435$.

Zhang XY, Fan F-M, Chen D-C, Tan Y-L, Tan S-P, Hu K et al (2016). Extensive white matter abnormalities and clinical symptoms in drug-naive patients with first-episode schizophrenia. J Clin Psychiatry 77: 205-211.

Supplementary Information accompanies the paper on the Neuropsychopharmacology website (http://www.nature.com/npp) 